УДК 697.329:006.354

\title{
СТРУКТУРЫ И ОПРЕДЕЛЯЮЩИЕ ПАРАМЕТРЫ ИМИТАЦИОННЫХ МОДЕЛЕЙ КОМПОНЕНТОВ ВЕТРОЭНЕРГЕТИЧЕСКИХ УСТАНОВОК ДЛЯ ПРОВЕРКИ УСТОЙЧИВОСТИ ЭНЕРГОСИСТЕМЫ
}

\author{
Андрианова Людмила Прокопьевна
}

д-р техн. наук, профессор

Байбурин Эдуард Рамилевич

канд. техн. наук, доцент

Уфимский государственный нефтяной

технический университет

Аннотация. В работе проведен анализ структурных схем и параметров имитационных моделей ряда компонентов ветроэнергетических установок (ВЭУ), присоединенных к энергосистеме. Представлены однопараметрическая и двухпараметрическая аэродинамические модели аэродинамического модуля, двухмассовая модель механического модуля; модели генераторов ВЭУ типов 3 и 4. Для двухпараметрической аэродинамической модели аэродинамического модуля приведено полное математическое описание и примеры аппроксимаций, которые можно использовать для определения параметров аэродинамической модели в конкретных вариантах при изготовлении ВЭУ. Приведена структура с описанием параметров, определяемых типом ВЭУ, для двухмассовой модели механического модуля. Приведены структура и параметры моделей генераторов для ВЭУ типа 3 с асинхронным генератором со статором, непосредственно подключаемым к энергосистеме, и ротором, подключаемым через электронный преобразователь; для ВЭУ типа 4 с синхронным и асинхронным генератором, подключаемым к энергосистеме через электронный преобразователь. Приведена структура и параметры модели вращения опорной системы отсчета. Приведены нормативные рекомендации производителям ВЭУ по определению параметров моделей модулей в зависимости от варианта моделей ВЭУ и разработчикам программного обеспечения для расчета начального состояния с учетом представленных изготовителем необходимых параметров.

Ключевые слова: ветроэнергетические установки, аэродинамический модуль, механический модуль, генератор, электрооборудование, имитационные модели, структура и параметры. 


\title{
STRUCTURES AND DEFINING PARAMETERS OF SIMULATION MODELS OF WIND POWER PLANT COMPONENTS FOR TESTING THE STABILITY OF THE POWER SYSTEM
}

\author{
Andrianova Lyudmila Prokopyevna \\ Doctor of Technical Sciences, Professor \\ Baiburin Eduard Ramilevich \\ Candidate of Technical Sciences \\ Associate Professor
}

\begin{abstract}
The paper analyzes the structural schemes and parameters of simulation models of a number of components of wind power plants (wind turbines) connected to the power system. One-parameter and two-parameter aerodynamic models of an aerodynamic module, a two-mass model of a mechanical module, and models of wind turbine generators of types 3 and 4 are presented. For a twoparameter aerodynamic model of an aerodynamic module, a complete mathematical description and examples of approximations that can be used to determine the parameters of the aerodynamic model in specific variants in the manufacture of wind turbines are given. The structure with the description of the parameters determined by the type of wind turbine for the two-mass model of the mechanical module is given. The structure and parameters of generator models for type 3 wind turbines with an asynchronous generator with a stator directly connected to the power system and a rotor connected via an electronic converter are given; for a type 4 wind turbine with a synchronous and asynchronous generator connected to the power system via an electronic converter. The structure and parameters of the rotation model of the reference frame are given. Regulatory recommendations are given to manufacturers of wind turbines for determining the parameters of module models, depending on the variant of wind turbine models, and to software developers for calculating the initial state, taking into account the necessary parameters provided by the manufacturer.
\end{abstract}

Key words: wind power plants, aerodynamic module, mechanical module, generator, electrical equipment, simulation models, structure and parameters.

Имитационные модели компонентов ветроэнергетических установок (ВЭУ) создают для проверки устойчивости энергосистемы при изменении базовых номинальных параметров ВЭУ при возникновении возмущений в 
энергосистеме и отклонении параметров ВЭУ при воздействии внешних климатических факторов, например, скорости ветра [1-5].

Аэродинамический модуль. Модели аэродинамического модуля:

1) Модель с постоянным аэродинамическим моментом;

2) Однопараметрическая аэродинамическая модель;

3) Двухпараметрическая аэродинамическая модель.

Модель с постоянным аэродинамическим моментом

Структурная схема модели аэродинамического модуля с постоянным аэродинамическим моментом показана на рис. 1.

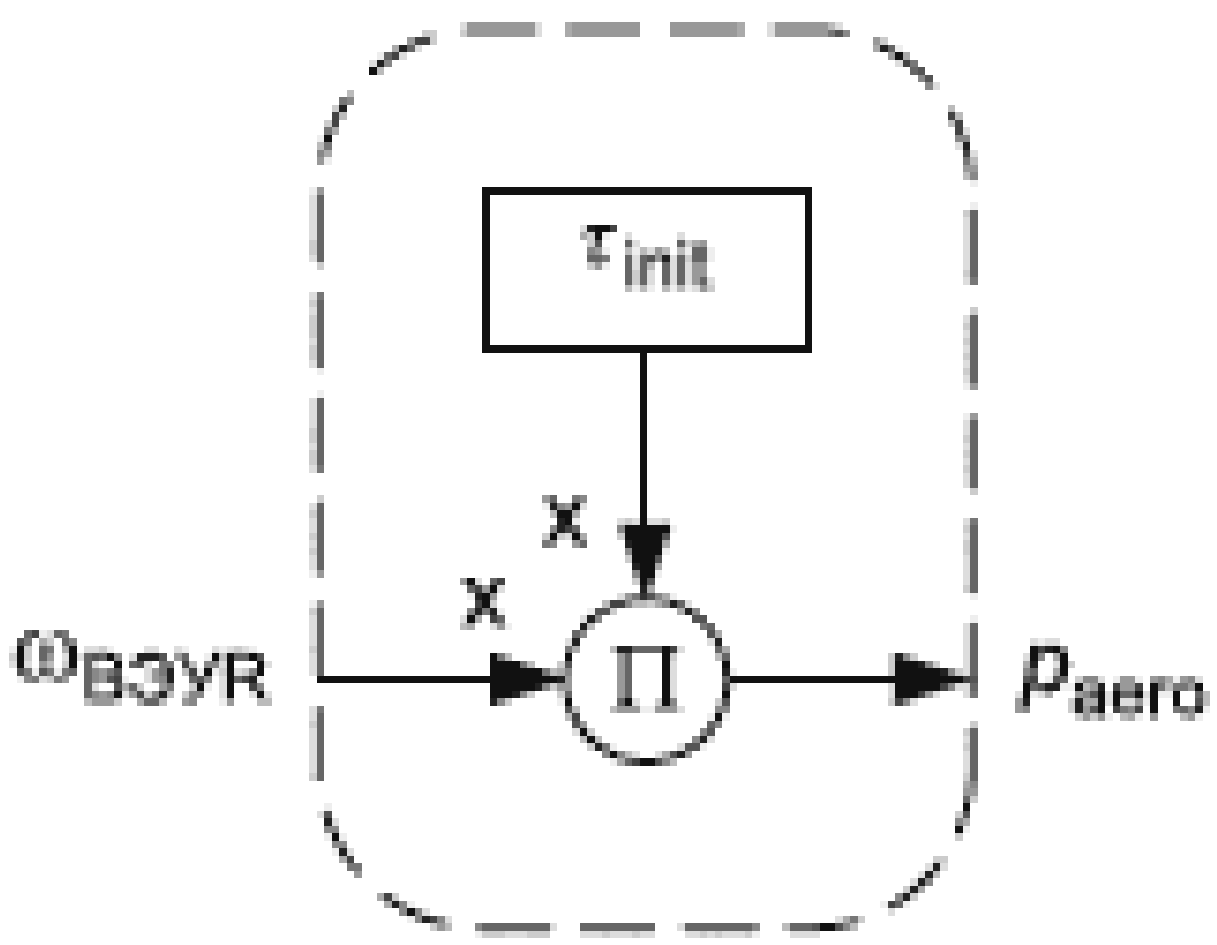

Рис. 1. Структурная схема модели с постоянным аэродинамическим моментом [4, с.30]: $\tau_{\text {init }}$ - начальный момент трансмиссии; $P_{\text {aero }} \quad$ - аэродинамическая мощность; $P_{\text {aero }} \quad$ ФвэуR $_{\text {в }}$ угловая скорость вращения ветроколеса ВЭУ

\section{Однопараметрическая аэродинамическая модель}

В однопараметрической аэродинамической модели учитывается зависимость мощности от угла установки лопастей, но пренебрегается зависимостью мощности от скорости вращения ветроколеса/ротора.

Для однопараметрической аэродинамической модели не требуется какихлибо определяемых изготовителем ВЭУ параметров. Начальный стационарный момент трансмиссии $\tau_{\text {init }}$ должен быть задан по параметрам 
потокораспределения мощности в соответствии с ГОСТ Р 54418.27.1 [4, рисунок 3, с.15 ].

Структурная схема модели показана на рис. 2, параметры модели приведены в таблице 1. Начальная активная мощность $p_{\text {init }}$ должна быть задана по параметрам потокораспределения мощности в соответствии с ГОСТ Р 54418.27.1 [4, рисунок 3, с.15 ].

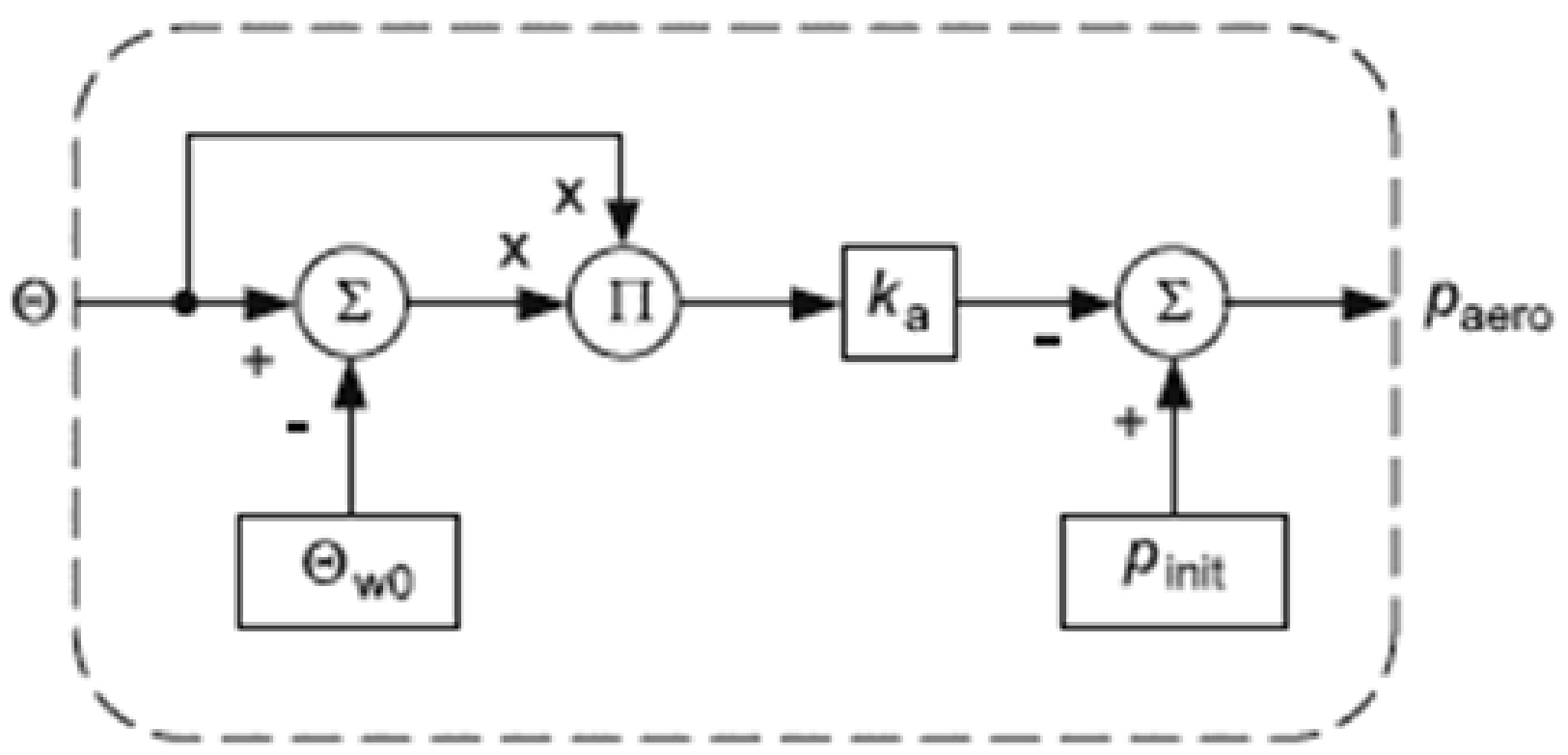

Рис. 2. Структурная схема однопараметрической аэродинамической модели $[4$, с.31]

Таблица 1

Параметры однопараметрической аэродинамической модели

\begin{tabular}{|c|c|l|}
\hline Обозначение & Базисная величина & \multicolumn{1}{|c|}{ Описание } \\
\hline$\Theta_{\mathrm{w0}}$ & град & $\begin{array}{l}\text { Начальный угол установки лопастей. } \\
\text { Определяется предшествующим } \\
\text { установившимся режимом ВЭУ }\end{array}$ \\
\hline$k_{\mathrm{a}}$ & $P_{\text {ном }} /$ град $^{2}$ & $\begin{array}{l}\text { Коэффициент аэродинамического усиления. } \\
\text { Определяется типом ВЭУ. }\end{array}$ \\
\hline
\end{tabular}

Двухпараметрическая аэродинамическая модель

Структурная схема двухпараметрической аэродинамической модели показана на рис. 3, параметры модели приведены в таблице 2. 


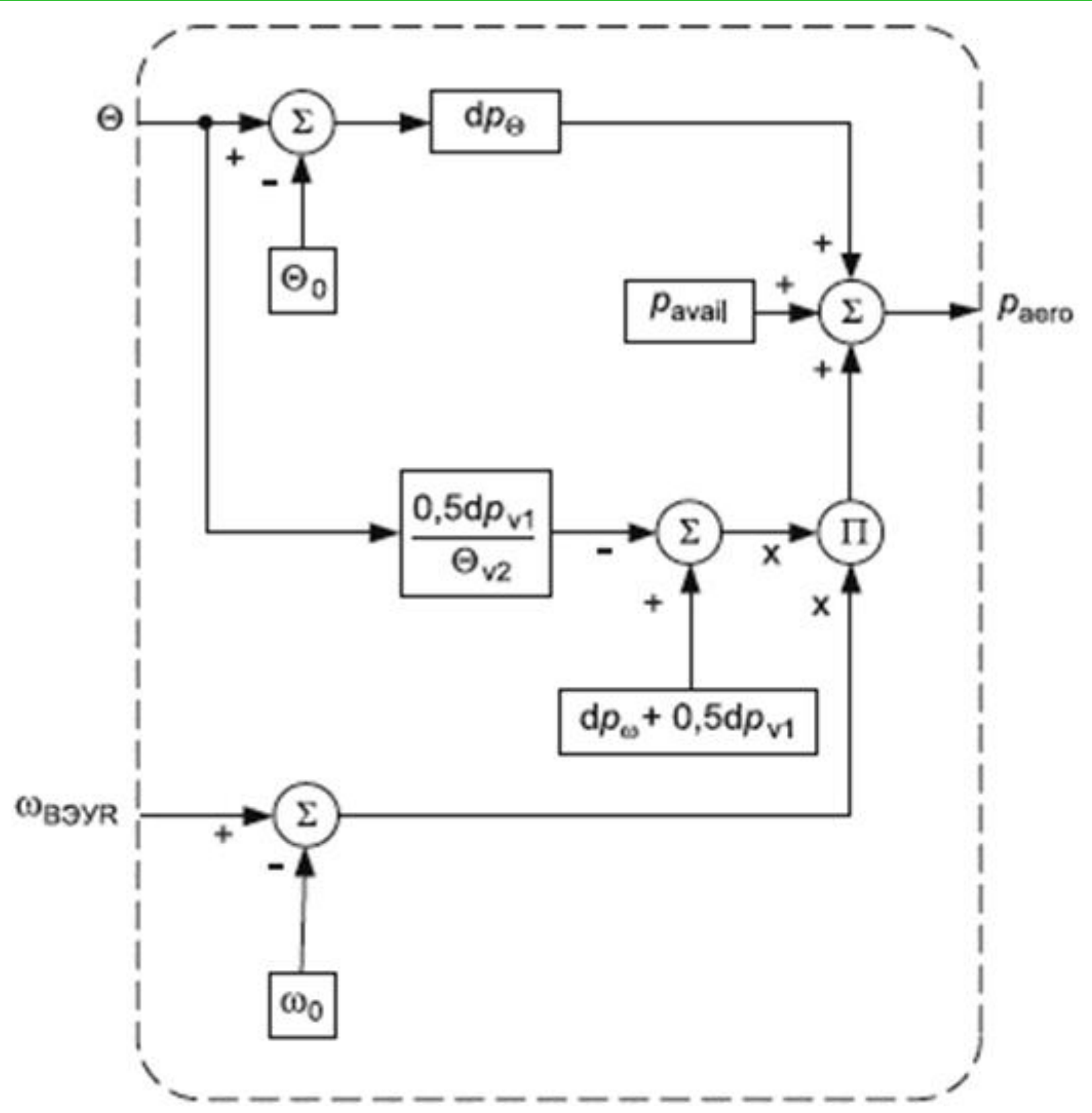

Рис. 3. Структурная схема двухпараметрической аэродинамической модели $[4, \mathrm{c.32}]$

Таблица 2

\section{Параметры двухпараметрической}

\section{аэродинамической модели}

\begin{tabular}{|c|c|l|}
\hline Обозначение & $\begin{array}{c}\text { Базисная } \\
\text { величина }\end{array}$ & \multicolumn{1}{c|}{ Описание } \\
\hline$p_{\text {avail }}$ & $P_{\text {ном }}$ & $\begin{array}{l}\text { Доступная аэродинамическая мощность. } \\
\text { Определяется предшествующим } \\
\text { установившимся режимом ВЭУ. }\end{array}$ \\
\hline
\end{tabular}




\begin{tabular}{|c|c|c|}
\hline$\Theta_{0}$ & град & 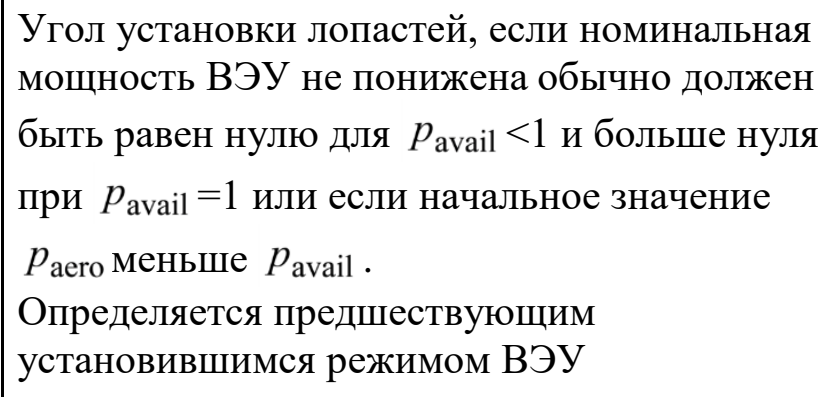 \\
\hline$\omega_{0}$ & $\Omega_{\text {base }}$ & $\begin{array}{l}\text { Скорость ветроколеса/ротора, если } \\
\text { номинальная мощность ВЭУ не понижена. } \\
\text { Определяется типом ВЭУ. }\end{array}$ \\
\hline $\mathrm{d} p_{\Theta}$ & $P_{\text {ном }} /$ град & $\begin{array}{l}\text { Частная производная аэродинамической } \\
\text { мощности по углу установки лопастей. } \\
\text { Определяется типом ВЭУ. }\end{array}$ \\
\hline $\mathrm{d} p_{\omega}$ & $P_{\text {ном }} / \Omega_{\text {base }}$ & $\begin{array}{l}\text { Частная производная аэродинамической } \\
\text { мощности по угловой скорости вращения } \\
\text { ветроколеса/ротора . } \\
\text { Определяется типом ВЭУ. }\end{array}$ \\
\hline$\Theta_{\mathrm{v} 2}$ & град & $\begin{array}{l}\text { Угол установки лопастей при удвоенной } \\
\text { номинальной скорости ветра. } \\
\text { Определяется типом ВЭУ. }\end{array}$ \\
\hline $\mathrm{d} p_{\mathrm{v} 1}$ & $P_{\text {ном }} / \Omega_{\text {base }}$ & $\begin{array}{l}\text { Частная производная } d p_{\omega} \text { при номинальной } \\
\text { скорости ветра. } \\
\text { Определяется типом ВЭУ. }\end{array}$ \\
\hline
\end{tabular}

Доступная аэродинамическая мощность $p_{\text {avail }}$ позволяет выполнить моделирование с пониженным номиналом для объединения с устройством управления мощностью ВЭС для того, чтобы было возможно возрастание мощности ВЭУ, если для этого достаточно аэродинамической мощности. Начальная аэродинамическая мощность не может превосходить $p_{\text {avail }}$.

Математическое описание модели [4, с. 77]

Аэродинамическая мощность ВЭУ $P_{\text {aero }}$ обычно рассчитывается по формуле

$$
P_{\text {aero }}=\frac{1}{2} \rho \pi R_{\text {ВЭуг }}^{2} V^{3} C_{\mathrm{p}}(\Lambda, \Theta),
$$

где $\rho$ - плотность воздуха;

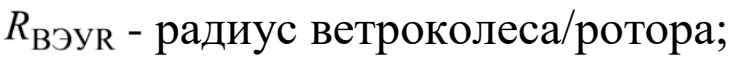

$V$ - скорость ветра; 
$C_{\mathrm{p}}(\Lambda, \Theta)$ - коэффициент мощности, который зависит от угла установки лопастей $\Theta и$ быстроходности ветроколеса/ротора $\Lambda$, определяемой по выражению

$$
\Lambda=\frac{R_{\mathrm{B} \ni \mathrm{YR}_{\mathrm{R}} \Omega_{\mathrm{BЭ \textrm {R }}}}}{V},
$$

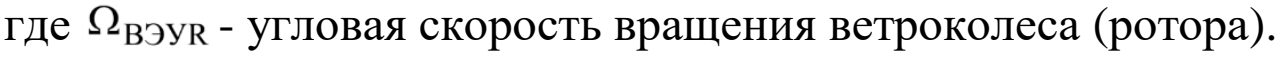

Аэродинамическая мощность в относительных единицах $p_{\text {aero }}\left(\mathrm{v}, \Theta, \omega_{\mathrm{R}}\right)$ является функцией скорости ветра в относительных единицах $\mathrm{v}$, угла установки лопастей $\Theta$ и угловой скорости вращения ветроколеса (ротора) в относительных единицах $\omega_{\mathrm{R}}$. В установившемся режиме, соответствующем скорости ветра $\mathrm{v}_{0}$, доступная аэродинамическая мощность $p_{\text {avail }}\left(\mathrm{v}_{0}\right)$ при угле установки лопастей $\Theta_{0}$ и угловой скорости вращения ветроколеса (ротора) $\omega_{0}$ определяется выражением

$$
p_{\text {avail }}\left(v_{0}\right)=p_{\text {aero }}\left(v_{0}, \Theta_{0}, \omega_{0}\right) .
$$

В предположении постоянной скорости ветра $\mathrm{v}_{0}$ ряд Тейлора задает линейное представление характеристик аэродинамической мощности в окрестности рабочего значения доступной аэродинамической мощности $\left(\mathrm{v}_{0}, \Theta_{0}, \omega_{0}\right)$

$$
p_{\text {aero }}=p_{\text {avail }}+\mathrm{d} p_{\Theta}\left(\Theta-\Theta_{0}\right)+\mathrm{d} p_{\omega}\left(\omega_{\mathrm{B} \ni \mathrm{yR}}-\omega_{0}\right) .
$$

Частные производные $\mathrm{d} p_{\Theta}$ и $\mathrm{d} p_{\omega}$ определяются по формулам:

$$
\begin{aligned}
& \mathrm{d} p_{\omega}=\left.\frac{\partial p_{\text {aero }}}{\partial \omega_{\text {ВЭув }}}\right|_{\nu=v_{0}, \Theta=\Theta_{0}}, \\
& \mathrm{~d} p_{\Theta}=\left.\frac{\partial p_{\text {aero }}}{\partial \Theta}\right|_{\nu=v_{0}, \Theta=\Theta_{0}} .,
\end{aligned}
$$

\section{Аппроксимация параметров модели}

Аэродинамическая модель, представленная выражением (4), должна использоваться с $\Theta$ и $\omega_{\mathrm{R}}$ в качестве входных величин и набором зависящих от варианта параметров $p_{\text {avail }}, \Theta_{0}, \omega_{0}, \mathrm{~d} p_{\Theta}$ И $\mathrm{d} p_{\omega}$.

Рассмотрим ряд аппроксимаций, которые изготовитель ВЭУ может использовать для определения параметров аэродинамической модели в 
конкретных вариантах.

На рис. 4, а приведена типовая номограмма рабочего режима ВЭУ в зависимости от угла установки лопастей и скорости ветра, на рис. 4, б представлена зависимость частной производной мощности по скорости вращения ветроколеса (ротора) $\partial p_{\text {aero }} / \partial \omega_{R}$ от угла установки лопастей и скорости ветра.

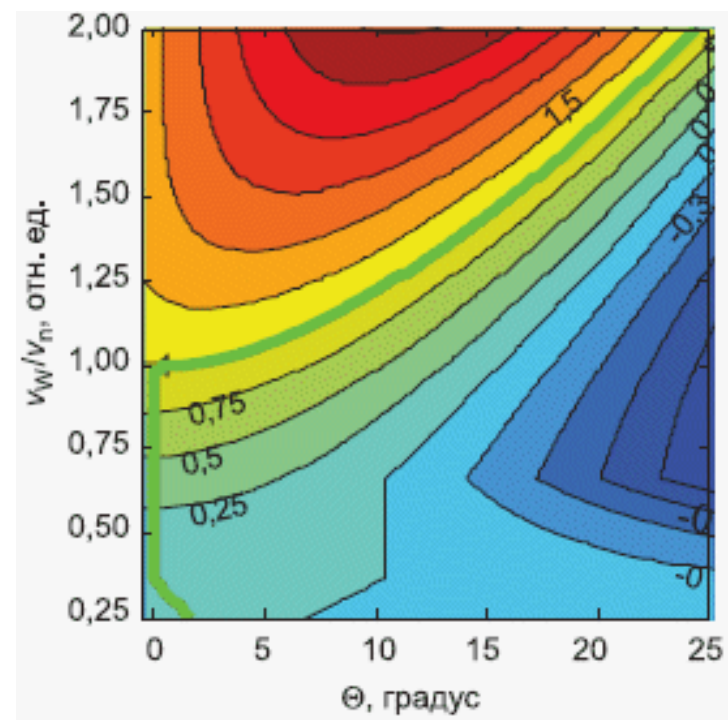

$\mathbf{a}$

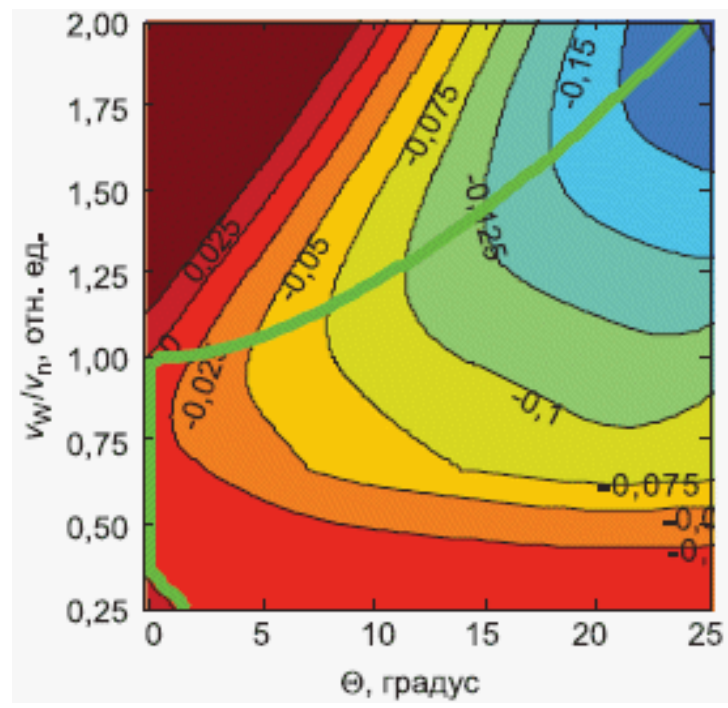

б

Рис. 4. Типовые номограммы рабочего режима ВЭУ [4, с. 78-79]: a - зависимость аэродинамической мощности от угла установки лопастей

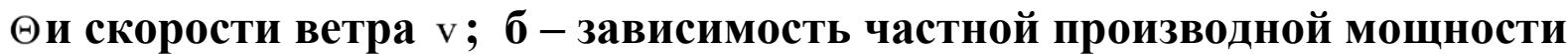
по угловой скорости вращения ветроколеса/ротора $\partial p_{\text {aero }} / \partial \omega_{\mathrm{R}}$ от угла установки лопастей $\Theta$ икорости ветра $v$

Как показано на рис. 5, а частную производную мощности по углу установки лопастей вдоль рабочей траектории на номограмме можно описать простым способом как линейную функцию двух параметров, представляющих наклон и смещение. Такое представление так же справедливо в случае работы ВЭУ при неполной мощности.

$$
\mathrm{d} p_{\Theta}(\Theta)=K_{\text {aero }} \Theta+C_{\text {aero . }}
$$

На рис. 5, б показана частная производная мощности по изменению скорости вращения ветроколеса/ротора вдоль рабочей траектории номограммы для работы при номинальной мощности и 0,5 от номинальной 
мощности.

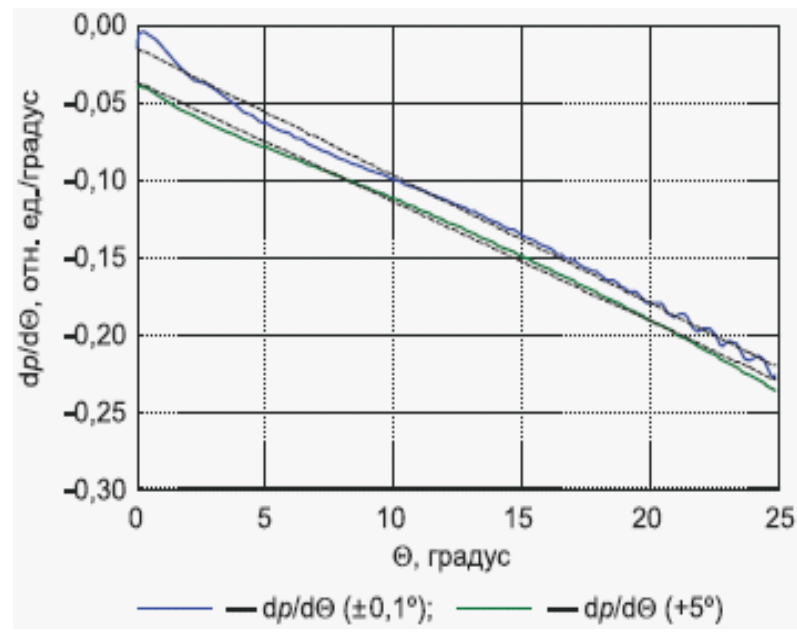

$\mathbf{a}$

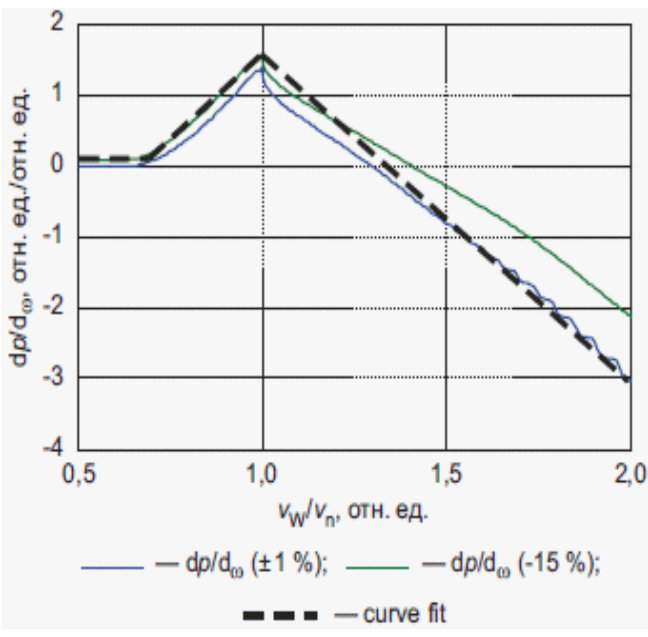

б

Рис. 5. Зависимость частной производной мощности [4, с. 79- 80]:

a - по углу установки лопастей от угла установки лопастей $\Theta$;

б - по изменению скорости вращения ветроколеса/ротора от скорости ветра при 1 (сплошная линия) и 0,5 (пунктирная линия) в относительных единицах активной мощности

Функциональная зависимость для номинальной мощности может быть аппроксимирована с помощью четырех параметров, указанных в таблице 3.

Таблица 3

Точки, характеризующие соотношение между скоростью ветра $v$ и частной производной $\mathrm{d} p_{\omega}[4, \mathrm{c.} 80]$

\begin{tabular}{|l|c|c|c|c|}
\hline Входное значение, $v$ & 0 & $v_{\omega 1}$ & 1 & 2 \\
\hline Выходное значение, $\mathrm{d} p_{\omega}$ & $\mathrm{d} p_{\omega 1}$ & $\mathrm{~d} p_{\omega 1}$ & $\mathrm{~d} p_{\mathrm{v} 1}$ & $\mathrm{~d} p_{\mathrm{v} 2}$ \\
\hline
\end{tabular}

Воздействие изменения угла установки лопастей на $\mathrm{d} p_{\omega}$ при снижении мощности (работа при части номинальной мощности) может быть аппроксимировано формулой

$$
\mathrm{fd} p_{v 1}(\Theta)=\frac{\mathrm{d} p_{v 1}}{2 \Theta_{v 2}}\left(\Theta_{v 2}-\Theta\right)
$$


Окончательное определение $\mathrm{d} p_{\omega}$ задается формулой

$$
\mathrm{d} p_{\omega}(v, \Theta)= \begin{cases}-\frac{\mathrm{d} p_{v 1}}{2}+\mathrm{d} p_{\omega 1} & \text { для } v \leq v_{\omega 1} \\ -\frac{\mathrm{d} p_{v 1}}{2}+\mathrm{d} p_{\omega 1}+\frac{v-v_{\omega 1}}{1-v_{\omega 1}}\left(\mathrm{~d} p_{v 1}-\mathrm{d} p_{\omega 1}\right) & \text { для } v_{\omega 1}<v \leq 1 \\ \frac{\mathrm{d} p_{v 1}}{2}+(v-1)\left(\mathrm{d} p_{v 2}-\frac{\mathrm{d} p_{v 1}}{2}\right) & \text { для } v>1\end{cases}
$$

Поскольку $\mathrm{d} p_{\omega}$ является функцией скорости ветра, требуется задать значение начальной скорости ветра. Зависимость между скоростью ветра $\boldsymbol{v}$ и соответствующей доступной аэродинамической мощностью определяется кривой $p_{\mathrm{pc}}(\mathrm{v})$. Кривая мощности определяется при помощи единственного параметра, который задает скорость ветра $v_{\mathrm{WP} 03}$ при значении активной мощности 0,3 (рис. 6, а). Ниже этого значения предполагается кубическая зависимость между скоростью ветра и мощностью, как показано в формуле (9). При более высоких скоростях ветра вплоть до номинальной, $v_{\mathrm{WP} 03} \leq v \leq 1$, используется линейная аппроксимация. В итоге доступная аэродинамическая мощность при скорости ветра определяется по следующей формуле:

$$
p_{\mathrm{pc}}(v)= \begin{cases}\frac{0,3}{v_{\mathrm{WPO} 3}^{3}} v^{3} & \text { для } 0<v \leq v_{\mathrm{WPO} 3} \\ 1+\frac{0,7}{1-v_{\mathrm{WPO} 3}}(v-1) & \text { для } v_{\mathrm{WPO} 3}<v \leq 1 \\ 1 & \text { для } v>1\end{cases}
$$

Отношение между скоростью ветра и углом установки лопастей определяется аппроксимацией

$$
\Theta_{0}\left(v_{0}\right)= \begin{cases}0 & \text { для } v_{0} \leq 1 \\ \Theta_{v 2} \frac{4}{3}\left(1-\frac{1}{v_{0}^{2}}\right) & \text { для } v_{0}<1,\end{cases}
$$

где $\Theta_{\mathrm{v} 2}-$ угол установки лопастей при удвоенной номинальной скорости 
ветра (рис. 6, б).

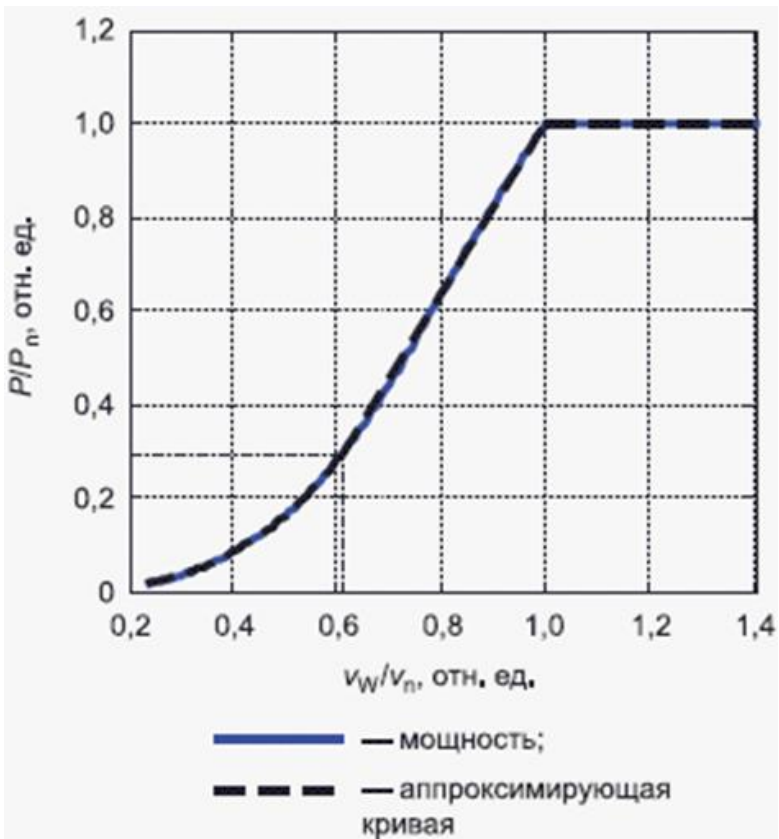

a

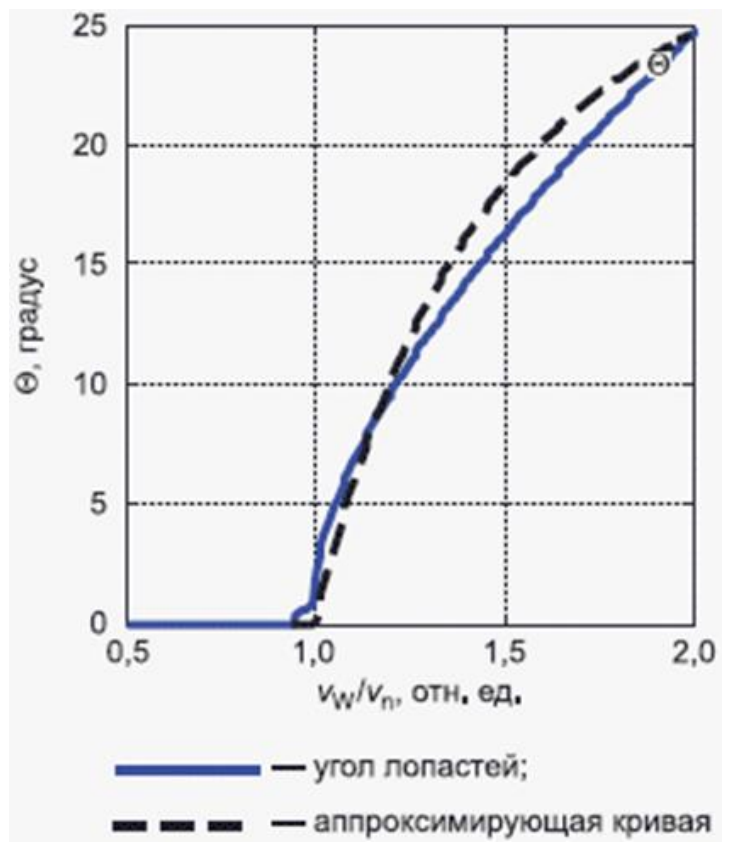

б

Рис. 6. Аппроксимация зависимостей параметров двухпараметрической аэродинамической модели от скорости ветра [4, с. 81-82]:

a - аэродинамической мощности; б - угла установки лопастей

Объединяя аппроксимации, представленные на рис. 6, изготовитель может описать аэродинамические характеристики конкретного варианта одного из типов ВЭУ при помощи параметров, приведенных в таблице 4.

Таблица 4

Параметры для описания аэродинамических характеристик ВЭУ конкретного типа $[4$, с. 82]

\begin{tabular}{|c|c|l|}
\hline Обозначение & $\begin{array}{c}\text { Базисная } \\
\text { величина }\end{array}$ & \multicolumn{1}{c|}{ Описание (формула) } \\
\hline $\mathrm{v}_{\text {wP03 }}$ & $\mathrm{V}_{\text {ном }}$ & Скорость ветра при 0,3 номинальной мощности $(9)$ \\
\hline$C_{\text {aero }}$ & $P_{\text {ном }} /$ град/ $/ V_{\mathrm{N}}$ & Смещение в линейном представлении $\partial p_{\Theta}(6)$ \\
\hline$K_{\text {aero }}$ & $P_{\text {ном }} /$ град & Наклон в линейном представлении $\partial p_{\Theta}(6)$ \\
\hline$v_{\omega 1}$ & $\mathrm{~V}_{\text {ном }}$ & $\begin{array}{l}\text { Скорость ветра, при которой достигается номинальная } \\
\text { скорость ветроколеса/ротора (в предположении } \\
\text { идеального управления) }(8)\end{array}$ \\
\hline $\mathrm{d} p_{\omega 1}$ & $P_{\text {ном }} / \Omega_{\text {base }}$ & $\begin{array}{l}\mathrm{d} p_{\omega} \text { при угловой скорости ветроколеса/ротора ниже } \\
\text { номинальной }(8)\end{array}$ \\
\hline $\mathrm{d} p_{v 2}$ & $P_{\text {ном }} / \Omega_{\text {base }}$ & $\begin{array}{l}\mathrm{d} p_{\omega} \text { при удвоенной номинальной угловой скорости } \\
\text { ветроколеса/ротора }(8)\end{array}$ \\
\hline
\end{tabular}


Корректное использование модели возможно только в условиях пониженной мощности, когда начальная мощность $p_{\text {init }} \leq p_{\text {avail }}$.

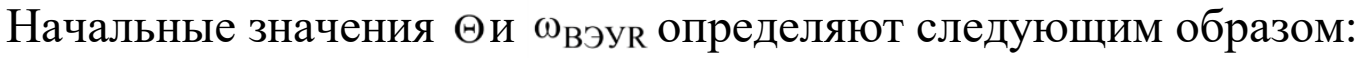

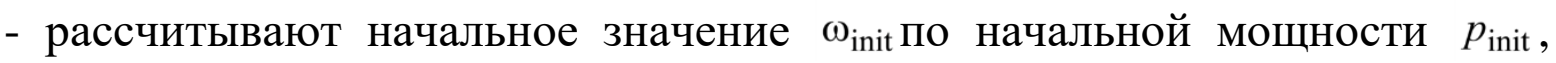
используя справочную таблицу зависимости угловой скорости вращения ветроколеса (ротора) от мощности $\omega=f(p)[4$, с. 44$]$;

- определяют начальное значение $\Theta_{\text {init }}$ по формуле

$$
\Theta_{\text {init }}=\Theta_{0}+\frac{p_{\text {init }}-p_{\text {wind }}-\mathrm{d} p_{\omega}\left(\omega_{\text {init }}-\omega_{0}\right)}{\mathrm{d} p_{\Theta}} .
$$

Модель механического модуля. Двухмассовая модель.

Структурная схема модели механического модуля показана на рис. 7, параметры модели приведены в таблице 5.

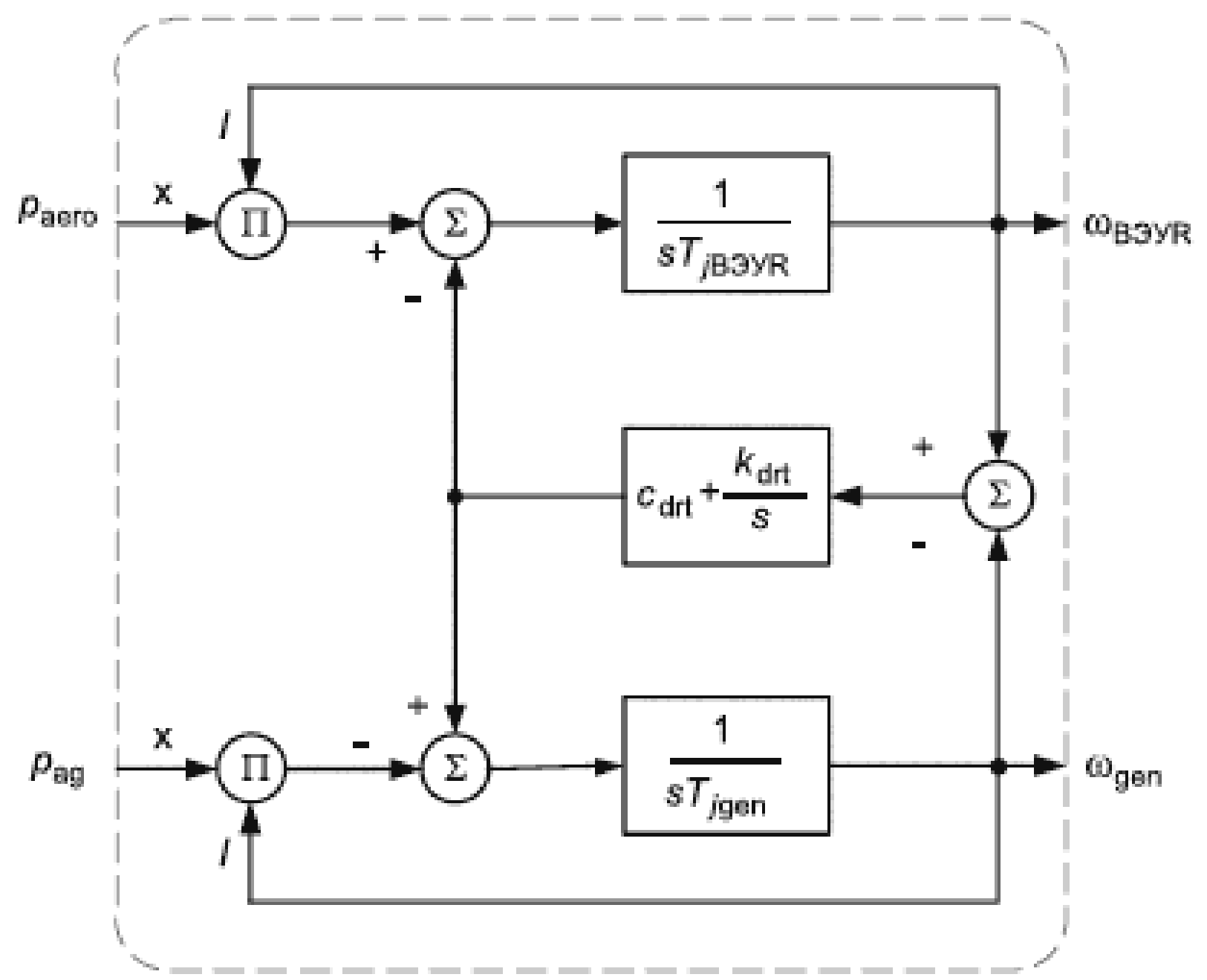

Рис. 7. Структурная схема двухмассовой модели [4, с.32 ] 
Таблица 5

\section{Параметры двухмассовой модели}

\begin{tabular}{|c|c|l|}
\hline Обозначение & Базисная величина & \multicolumn{1}{|c|}{ Описание } \\
\hline$T_{j \text { вэур }} *$ & $\mathrm{c}$ & Постоянная инерции ветроколеса/ротора \\
\hline$T_{j \text { gen }} *$ & $\mathrm{c}$ & Постоянная инерции генератора \\
\hline$k_{\mathrm{drt}}$ & $M_{\text {base }}$ & Коэффициент жесткости трансмиссии \\
\hline$c_{\mathrm{drt}}$ & $M_{\text {base }} / \Omega_{\text {base }}$ & Коэффициент затухания колебаний трансмиссии \\
\hline
\end{tabular}

Параметры двухмассовой модели $T_{j \text { вэур }}, T_{j \mathrm{gen}}, k_{\mathrm{drt}}, c_{\mathrm{drt}}$ определяются типом ВЭУ. Некоторые программные средства включают учет инерции генератора во встроенную модель генератора. В этом случае дополнительная механическая модель должна взаимодействовать с валом генератора вместо воздушного зазора генератора и, следовательно, не включать инерцию генератора. Полная механическая модель при этом остается двухмассовой моделью [4].

\section{Модель генератора ВЭУ типа 3А}

Структурная схема модели показана на рис. 8, а параметры модели приведены в таблице 6.

Таблица 6

\section{Параметры модели генератора ВЭУ типа 3А}

\begin{tabular}{|c|c|l|}
\hline Обозначение & $\begin{array}{c}\text { Базисная } \\
\text { величина }\end{array}$ & \multicolumn{1}{|c|}{ Описание } \\
\hline$K_{P c}$ & - & $\begin{array}{l}\text { Коэффициент передачи общего канала регулирования } \\
\text { ПИ-регулятора тока }\end{array}$ \\
\hline$T_{I c}$ & $\mathrm{c}$ & Постоянная времени интегрального канала ПИ-регулятора тока \\
\hline$x_{\mathrm{S}}$ & $Z_{\text {base }}$ & Электромагнитное переходное индуктивное сопротивление \\
\hline $\mathrm{d} i_{\mathrm{pmax}}$ & $I_{\text {ном }} / \mathrm{c}$ & Максимальная скорость нарастания активного тока \\
\hline $\mathrm{d} i_{\text {qmax }}$ & $I_{\text {ном }} / \mathrm{c}$ & Максимальная скорость нарастания реактивного тока \\
\hline
\end{tabular}




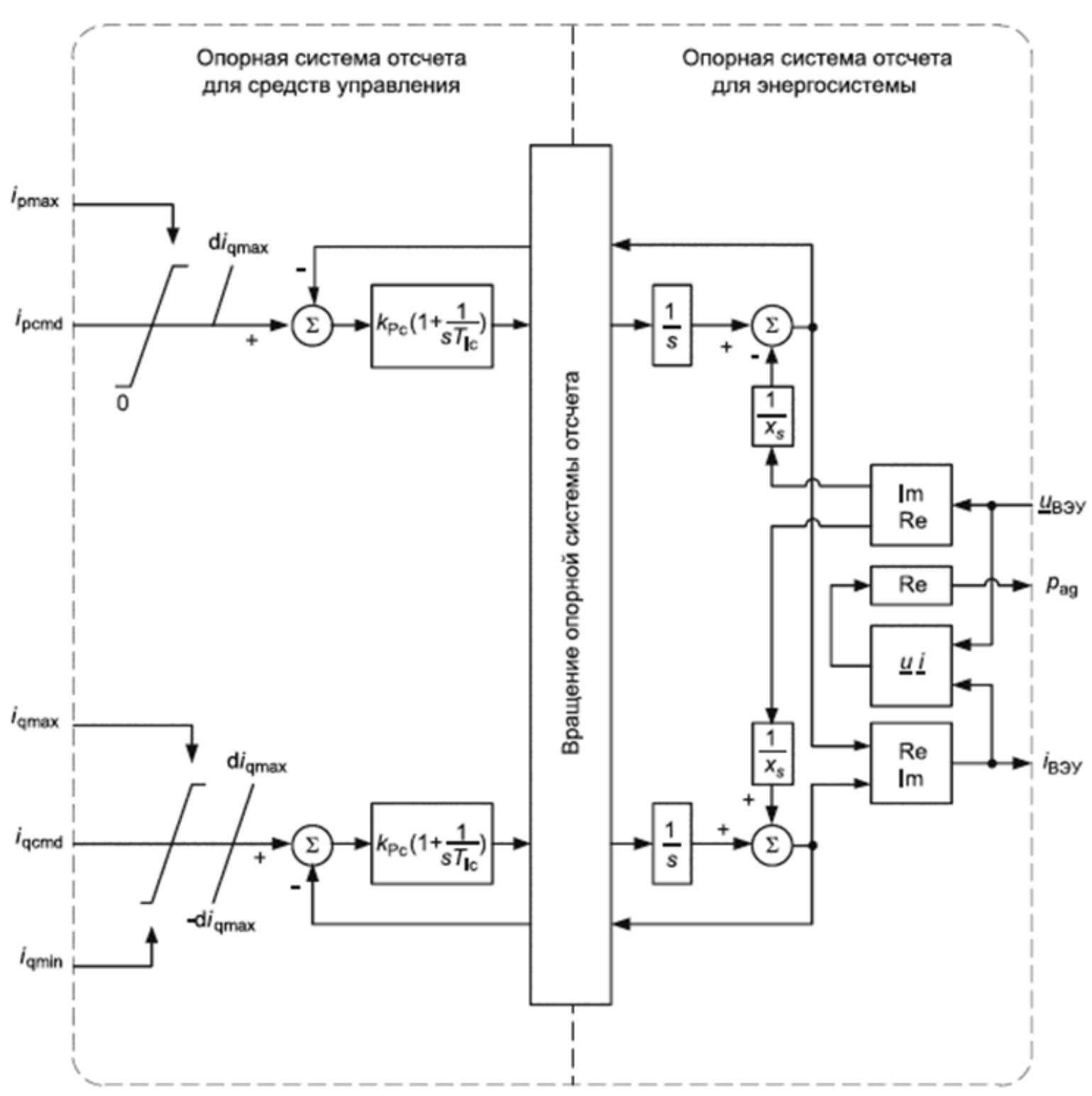

Рис. 8. Структурная схема модели генератора ВЭУ типа 3А:

$\operatorname{Re}$ - активное значение (действительное);

Im - реактивное значение (мнимое) $[4$, с. 34]

Параметры модели $K_{P c}, T_{I c}, x_{\mathrm{S}}$ определяются типом ВЭУ, параметры $\mathrm{d} i_{\text {pmax }}, \mathrm{d} i_{\text {qmax }}-$ проектом, т.е. работой ВЭУ в конкретной ВЭС.

Выходным параметром модели генератора является ток, выдаваемый в сеть регулируемым источником, который зашунтирован (поперечным) индуктивным сопротивлением $X_{\mathrm{s}}$. Однако в некоторых программных средствах моделирования компоненты управления и энергосистемы трактуются различно. Для улучшения надежности расчетных алгоритмов может оказаться целесообразным учесть шунтирующее сопротивление в уравнениях сети. 
Потерями в системе генератора пренебрегают, полагая мощность в воздушном зазоре между статором и ротором генератора $p_{a g}$ равной мощности на выводах ВЭУ.

\section{Модель звена вращения опорной системы отсчета}

Структурная схема модели показана на рис. 9, параметры модели приведены в таблице 7.

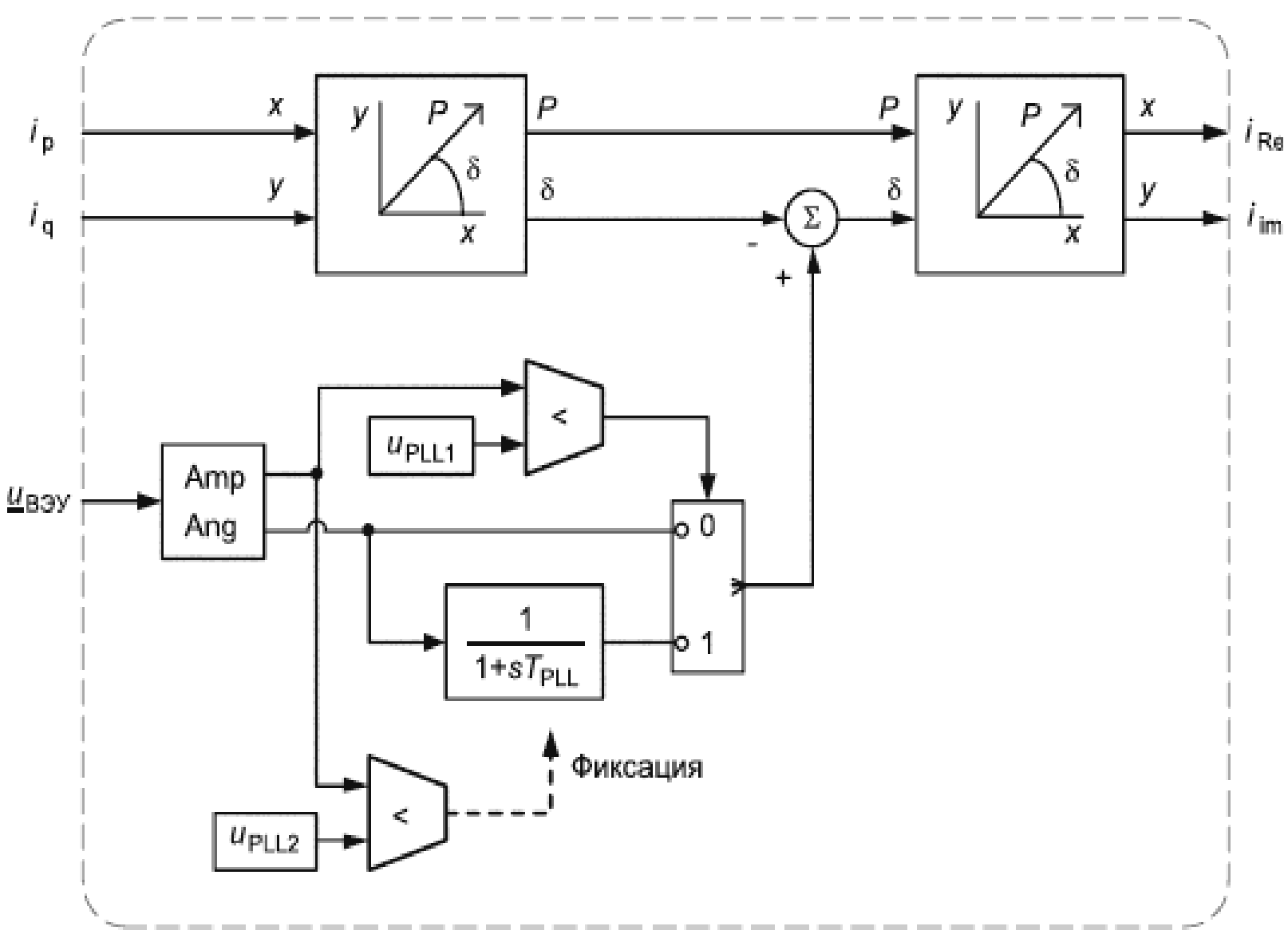

Рис. 9. Структурная схема модели вращения опорной системы отсчета:

Amp - амплитуда; Ang - угол [4, с. 38]

Таблица 7

Параметры модели вращения опорной системы отсчета

\begin{tabular}{|c|c|l|}
\hline Обозначение & $\begin{array}{c}\text { Базисная } \\
\text { величина }\end{array}$ & \multicolumn{1}{|c|}{ Описание } \\
\hline$T_{\mathrm{PLL}}$ & $\mathrm{c}$ & $\begin{array}{l}\text { Постоянная времени модели фильтра первого порядка модуля } \\
\text { автосинхронизации фазы }\end{array}$ \\
\hline
\end{tabular}




\begin{tabular}{|c|c|c|}
\hline$u_{\text {PLL1 }}$ & $U_{\text {ном }}$ & $\begin{array}{l}\text { Напряжение, ниже которого угол } \delta \text { отфильтрован и возможно } \\
\text { зафиксирован во избежание неустойчивости при пропадании } \\
\text { опорного напряжения. }\end{array}$ \\
\hline$u_{\text {PLL2 }}$ & $U_{\text {ном }}$ & $\begin{array}{l}\text { Напряжение, ниже которого угол } \delta \text { зафиксирован, если } \\
u_{\mathrm{PLL} 2} \leq u_{\mathrm{PLL} 1} \\
\text { Значение } u_{\mathrm{PLL} 2} \text { должно быть согласовано со значением } u_{\mathrm{PLL1}} \text { · } \\
\text { Обычно } u_{\mathrm{PLL} 2} \leq u_{\mathrm{PLL1}} \text {. } \\
\text { Напряжение } u_{\mathrm{PLL} 2 \text { применяется для того, чтобы избежать }} \\
\text { некорректности при вычислении, когда напряжение близко к } \\
\text { нулю и вследствие этого численное значение угла не } \\
\text { определено. }\end{array}$ \\
\hline
\end{tabular}

Параметры модели $T_{\mathrm{PLL}}, u_{\mathrm{PLL} 1}, u_{\mathrm{PLL} 2}$ определяются типом ВЭУ.

Модель генератора ВЭУ типа 3В

Структурная схема модели показана на рис. 10, параметры и режимы модели приведены в таблице 8.

Таблица 8

Параметры и режимы модели генератора ВЭУ типа 3В

\begin{tabular}{|c|c|l|}
\hline Обозначение & Базисная величина & \multicolumn{1}{|c|}{ Описание } \\
\hline$T_{g}$ & $\mathrm{c}$ & Постоянная времени генератора \\
\hline $\mathrm{d} i_{\text {pmax }}$ & $\mathrm{I}_{\text {ном }} / \mathrm{c}$ & $\begin{array}{l}\text { Максимальная скорость нарастания активного } \\
\text { тока }\end{array}$ \\
\hline $\mathrm{d} i_{\mathrm{qmax}}$ & $\mathrm{I}_{\text {ном }} / \mathrm{c}$ & $\begin{array}{l}\text { Максимальная скорость нарастания реактивного } \\
\text { тока }\end{array}$ \\
\hline$x_{\mathrm{S}}$ & $Z_{\text {bаse }}$ & Электромагнитная переходная реактивность \\
\hline$T_{C W}(\mathrm{~d} u)$ & с от $U_{\text {ном }}$ & $\begin{array}{l}\text { Зависимость длительности замыкания от } \\
\text { изменения напряжения (например, по справочной } \\
\text { таблице) }\end{array}$ \\
\hline$T_{\text {шо }}$ & с & $\begin{array}{l}\text { Постоянная времени сглаживающего фильтра } \\
\text { замыкающего устройства }\end{array}$ \\
\hline$M_{\text {Вэусwр }}$ & - & Режим управления замыкающего устройства \\
\hline
\end{tabular}

Параметры $T_{g}, x_{\mathrm{S}}$ определяются типом ВЭУ, $\mathrm{d} i_{\mathrm{pmax}}, \mathrm{d} i_{\mathrm{qmax}},-$ проектом,

т.е. работой ВЭУ в конкретной $\mathrm{BЭС,} \quad T_{C W}(\mathrm{~d} u), \quad T_{\text {wo }}, \quad M_{\text {ВЭу }} \mathrm{y}_{\text {cwp }} \quad-$ предшествующим установившимся режимом ВЭУ. 


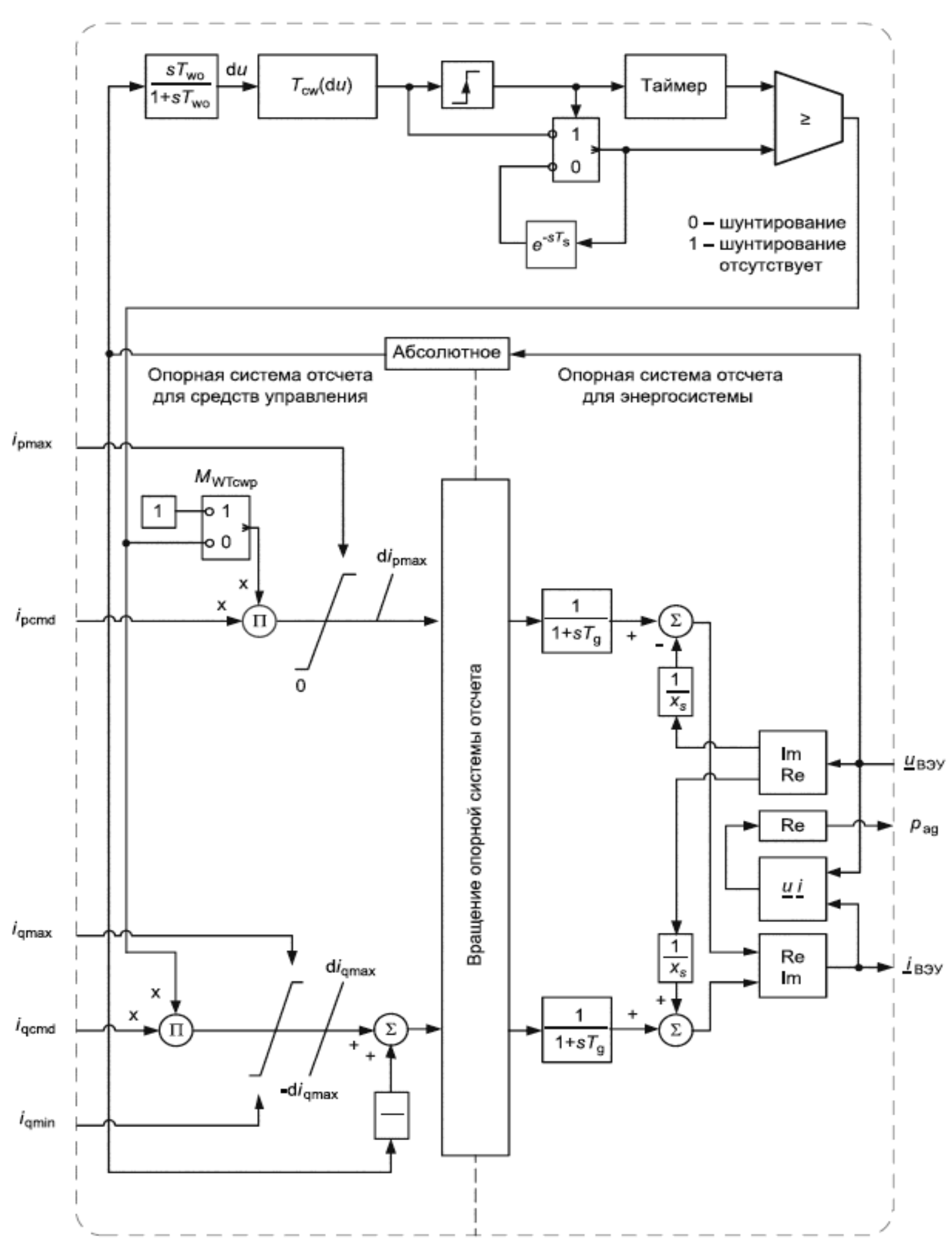

Рис. 10. Структурная схема модели генератора ВЭУ

типа 3В [4, с.36]

Эта модель является упрощением модели генератора ВЭУ типа 3А с добавлением модели замыкающего устройства. Выходным параметром модели генератора является ток. 
Однако в некоторых программных средствах моделирования для улучшения сходимости модели в модель модуля генератора необходимо добавить параллельное сопротивление $X_{\mathrm{s}}$. Для улучшения сходимости модели в таких случаях рекомендуется разместить это параллельное сопротивление, равное результату деления напряжения статора на переходную реактивность, так, чтобы оно было включено в уравнение энергосистемы.

\section{Модель генератора ВЭУ типа 4}

Структурная схема модели показана на рис. 11, параметры модели приведены в таблице 9.

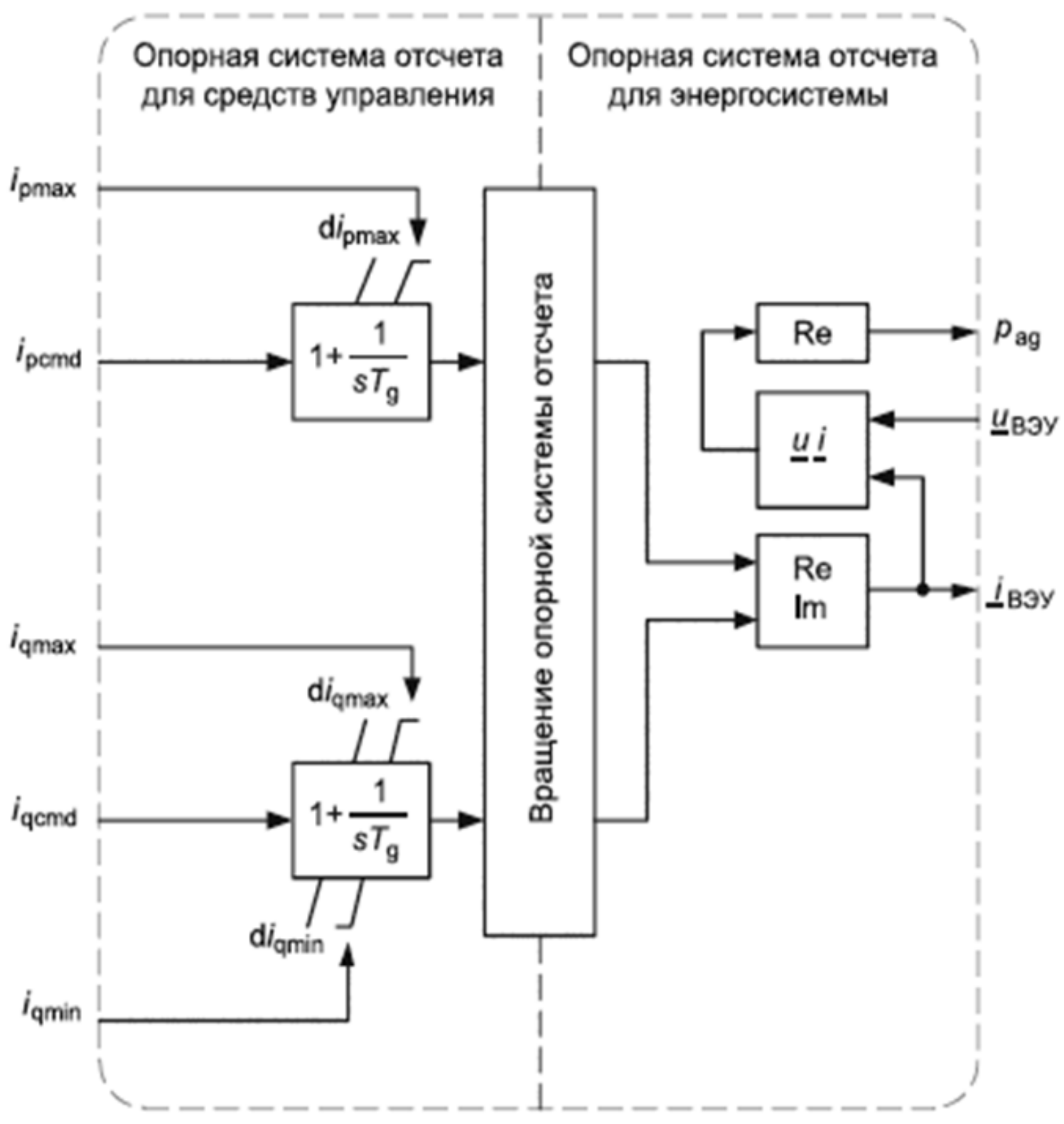

Рис. 11. Структурная схема модели генератора ВЭУ типа $4[4$, с.37] 
Таблица 9

\section{Параметры модели генератора ВЭУ типа 4}

\begin{tabular}{|c|c|l|}
\hline Обозначение & Базисная величина & \multicolumn{1}{|c|}{ Описание } \\
\hline$T_{g}$ & $\mathrm{c}$ & Постоянная времени генератора \\
\hline $\mathrm{d} i_{\text {pmax }}$ & $I_{\text {ном }} / \mathrm{c}$ & Максимальная скорость нарастания активного тока \\
\hline $\mathrm{d} i_{\mathrm{qmax}}$ & $I_{\text {ном }} / \mathrm{c}$ & Максимальная скорость нарастания реактивного тока \\
\hline $\mathrm{d} i_{\mathrm{qmin}}$ & $I_{\text {ном }} / \mathrm{c}$ & Минимальная скорость нарастания реактивного тока \\
\hline
\end{tabular}

Параметр модели $T_{g}$ определяется типом ВЭУ, параметры $\mathrm{d} i_{\mathrm{pmax}}, \mathrm{d} i_{\mathrm{qmax}}$, $\mathrm{d} i_{\text {qmin }}$ определяются проектом, т.е. работой ВЭУ в конкретной ВЭС.

При имитационном моделировании следует использовать стандартную модель асинхронного генератора. На практике при изучении устойчивости генератора является учет переходных процессов в электрической цепи ротора и пренебрежение переходными процессами в электрической цепи статора.

Модели электрооборудования [4, с. 39]:

\section{1) Модели шунтирующего конденсатора}

Для батарей конденсаторов с механическим переключением следует использовать стандартную модель конденсатора основной частоты, имеющуюся в средствах моделирования. Для батарей конденсаторов с тиристорным переключением может использоваться стандартная модель установки статической компенсации реактивной мощности;

\section{2) Модель автоматического выключателя}

При моделировании следует использовать стандартную модель автоматического выключателя, имеющуюся в средствах моделирования. Модель автоматического выключателя должна отключать выключатель при получении ею команды на отключение $\left[F_{\mathrm{OCB}}\right]$;

\section{3) Модель трансформатора}

Для моделирования следует использовать стандартную модель трансформатора, имеющуюся в средствах моделирования.

\section{Заключение}

Варианты проверки устойчивости энергосистемы характеризуются доступной аэродинамической мощностью $p_{\text {avail }}$. $\mathrm{B}$ таких вариантах проводится моделирование с пониженной мощностью, при котором на ВЭУ поступает сигнал опорной мощности $p_{\text {ref }} \leq p_{\text {avail }}$ от регулятора ВЭС, 
участвующей в регулировании частоты. Это может быть достаточным для единственного варианта с максимальной доступной аэродинамической мощностью при $p_{\text {avail }}=1$.

В вариантах, когда доступная аэродинамическая мощность $p_{\text {avail }}<1$, при изучении вариантов устойчивости в зависимости от доступной

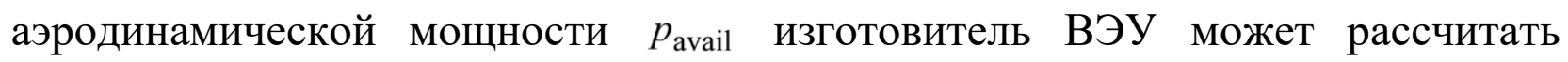
параметры, зависящие от варианта модели ВЭУ, следующим образом:

- если $p_{\text {avail }}=1$, выбирают скорость ветра $v_{0}>1$;

- если $p_{\text {avail }}<1, v_{0}$ может быть рассчитана по кривой мощности в (9), полагая $p_{\mathrm{pc}}\left(v_{0}\right)=p_{\text {avail }}$;

- рассчитывают $\mathrm{d} p_{\Theta}=\mathrm{d} p_{\Theta}\left(v_{0}\right)$ по формуле (6);

- рассчитывают $\mathrm{d} p_{\omega}=\mathrm{d} p_{\omega}\left(v_{0}\right)$ по формуле (8);

- рассчитывают $\omega_{0}$ по доступной аэродинамической мощности $p_{\text {avail }}$, используя справочную таблицу зависимости угловой скорости вращения ветроколеса (ротора) от мощности $\omega=f(p)$;

- определяют начальный угол установки лопастей $\Theta_{0}=\Theta_{0}\left(v_{0}\right)$ по формуле (10).

\section{Список литературы}

1. ГОСТ Р 51237-98 Нетрадиционная энергетика. Ветроэнергетика. Термины и определения. Введ. 01.07.1999; акт. 01.01.2021. - Москва: ГОССТАНДАРТ РОССИИ, 1999. - 16 с.

2. ГОСТ Р 55589-2013 (МЭК 60050-415:2999) Международный электротехнический словарь. Часть 415. Установки ветроэнергетические. Системы генерирования электроэнергии. Введ. 01.07.2014; акт. 01.01.2021. Москва: Стандартинформ, 2014. - 20 с.

3. ГОСТ Р 54418.1-2012 (МЭК 61400 -1:2005) Возобновляемая энергетика. Ветроэнергетика. Установки ветроэнергетические. Часть 1. Технические требования. Введ. 20.09.2012. - Москва: Стандартинформ, 2016. -88 c.

4. ГОСТ Р 54418.27.1-2019 (МЭК 61400-27-1:2015) Возобновляемая энергетика. Ветроэнергетика. Установки ветроэнергетические. Часть 27-1. Общие имитационные модели ветроэнергетических установок, присоединенных к энергосистеме. Введ. 01.06.2020; акт. 01.01.2021. - Москва: 
Стандартинформ, 2020. - 99 с.

5. ГОСТ Р 54418.21-2019 (МЭК 61400-21:2008) Возобновляемая энергетика. Ветроэнергетика. Установки ветроэнергетические. Часть 21. Измерение и оценка характеристик, связанных с качеством электрической энергии, ветроэнергетических установок, подключенных к электрической сети. Введ. 28.09.2011; акт. 01.01.2021. - Москва: Стандартинформ, 2012. - 50 с.

(C) Л.П. Андрианова, Э.Р. Байбурин, 2021 